\title{
Reparación de lesión condral patelar en un paciente pediátrico
}

\section{Repair of patellar chondral lesion in pediatric patient}

\author{
Pablo Tarazona Velutini, ${ }^{*}$ Alberto Azcona Cervera, ${ }^{*}$ Ricardo Velutini Becker*
}

\begin{abstract}
RESUMEN
Las lesiones condrales cada día son más frecuentes. Si éstas no son tratadas de manera adecuada, pueden causar secuelas graves. Se presenta el caso de un paciente de 15 años con una lesión condral grado 3 de acuerdo con la clasificación modificada de Outerbridge en la rótula, secundaria a una luxación femororrotuliana postraumática. Se realizó la reparación del ligamento patelofemoral medial y se reimplantó el fragmento condral mediante anclas con sutura sobre una base de un coágulo. El desenlace a los cuatro meses de seguimiento fue satisfactorio, pues el paciente no mostró dolor y sus arcos de movilidad estuvieron completos. El objetivo de este informe es presentar un caso clínico de un paciente con una lesión en el cartílago de la rótula, su manejo y los resultados postoperatorios.
\end{abstract}

Palabras clave: Lesión condral, rótula, reparación condral.

Nivel de evidencia: IV

\section{INTRODUCCIÓN}

La incidencia de las lesiones condrales varía de acuerdo con la edad: de 0.5 por cada 1,000 niños en edades de cuatro a cinco años y de 4.8 por cada 1,000 en niños de 12 a 13 años, presentando una mayor frecuencia en el sexo masculino que en el fe-

\footnotetext{
* Traumatología y Ortopedia del Centro Médico ABC
}

Recibido para publicación: 10/02/2019. Aceptado: 31/05/2019.

\section{Correspondencia:}

Dr. Alberto Azcona Cervera

Prol. Vasco de Quiroga Núm. 4001, Torre A, Piso 7, Consultorio 702,

Col. Santa Fe, 05370, Alcaldía Cuajimalpa, CDMX.

Teléfono: 81647268

E-mail: azconaortopedia@gmail.com

\begin{abstract}
Chondral lesions are more frequent every day, if not treated properly, they can cause serious sequelae. We present the case of a 15-year-old male patient with grade 3 modified Outerbridge chondral injury on the patella, secondary to a traumatic patellar dislocation. Medial patellofemoral ligament repair was performed and the chondral fragment was reimplanted with suture anchors on a clot base. At fourt months of follow-up. The outcome has been satisfactory results without pain, and with complete range of motion. The aim of this report is to present a clinical case of a patient with a cartilage injury, its treatment and postoperative results.
\end{abstract}

Keywords: Chondral lesion, patella, chondral repair.

Level of evidence: IV

menino. ${ }^{1}$ En la rótula, el mecanismo de lesión más común se debe a fuerzas cizallantes, lo que ocasiona una luxación femororrotuliana; además presenta una mayor incidencia en niños que en adultos..$^{2,3} \mathrm{Se}$ estima que las luxaciones de la rótula están asociadas con fracturas osteocondrales rotulianas hasta en $76 \%$; de éstas, $96 \%$ tienen rotura del ligamento patelofemoral medial. ${ }^{4}$

Los signos y síntomas dependen del grado de la lesión, a saber: dolor al palpar la rótula, signo de cepillo positivo, arcos de movilidad dolorosos, imposibilidad de apoyar el miembro pélvico afectado, bloqueo articular (si el fragmento actúa como cuerpo libre en rodilla) y aumento de volumen. ${ }^{5}$

El estudio diagnóstico no invasivo con mayor sensibilidad y especificidad es la resonancia magnética contrastada; sin embargo se puede realizar una resonancia magnética simple, la cual muestra 
buenos resultados. ${ }^{6,7}$ Las clasificaciones más usadas para las lesiones cartilaginosas son la de Outerbridge modificada ${ }^{2,8}$ y de la International Cartilage Repair Society (ICRS). ${ }^{8,9}$

El tratamiento puede variar de acuerdo con la extensión de la lesión. Así, las grado 1 y 2, de acuerdo con la clasificación de Outerbridge, generalmente se tratan con antiinflamatorios, reposo y fisioterapia; en tanto las lesiones 3 a 5 , se sugiere que se traten quirúrgicamente. ${ }^{10-12}$

El cartílago carece de propiedades regenerativas, ya que, ante alguna lesión, se sustituye el cartílago hialino por un fibrocartílago, ${ }^{13}$ lo que en teoría disminuirá la concentración de los proteoglicanos (queratán sulfato y condroitín sulfato). Esto incrementa el contenido de agua condral, lo que resulta en una disminución de la rigidez del cartílago y aumenta las fuerzas de transmisión de la carga al hueso subcondral, que es el causante del dolor. ${ }^{9,14}$ Por esta razón, existe controversia sobre cuál tratamiento es el adecuado para las lesiones en las que hay pérdida total del cartílago. ${ }^{15-17}$ Las técnicas más comúnmente utilizadas son el desbridamiento con microfracturas, el trasplante condral alógeno y la mosaicoplastia. ${ }^{16,18,19}$ Sin embargo, estas técnicas pueden tener resultados no favorables debido a la alteración de la biomecánica del cartílago, lo cual puede evolucionar con dolor, limitación de la movilidad y artrosis. ${ }^{20,21}$

\section{CASO CLÍNICO}

Presentamos el caso de un hombre de 15 años, quien acudió a consulta 24 horas después de caer desde su propia altura, cuyo impacto fue sobre su rodilla derecha, lo que causó una luxación de la articulación patelofemoral que se redujo en el sitio de la lesión. Después del traumatismo, el paciente presentó dolor intenso de 9/10 en la escala verbal análoga de dolor, el cual limitaba la marcha y se acompañaba de bloqueo articular.

$\mathrm{Al}$ momento de la exploración física, se encontró: aumento del volumen de la rodilla derecha, dolor al palpar la rótula en el borde medial y los arcos de movilidad estaban abolidos por el dolor; la maniobra de cepillo, a la palpación, era dolorosa; bostezos negativos; cajón anterior y posterior negativo, y maniobra de McMurray no valorable por dolor al intentar flexión de la rodilla. Se le indicó marcha con muletas sin apoyo, crioterapia, AINE y analgésicos. Se le realizó una resonancia magnética de rodilla derecha en la que se encontró rotura del ligamento patelofemoral medial, lesión grado 3 según la clasificación modificada de Outerbridge en la región central patelar de $2.3 \times 1.8 \mathrm{~cm}$. y fragmento libre en receso lateral (Figura 1 A y B).

Dos semanas después de la primera valoración, se le realizó una artrotomía con reparación dinámica del ligamento patelofemoral medial con injerto alógeno de tibial anterior y con dos anclas bioabsorbibles de $2.0 \mathrm{~mm}$ (Figura $2 \mathrm{~A}$ ). Al paciente también se le realizó una artrotomía mediante abordaje pararrotuliano medial, encontrando una lesión central del cartílago rotuliano (Figura 2B). Para ello, se localizó el fragmento condral, el cual estaba edematizado por el líquido articular, con un tamaño de $2.5 \times 2 \mathrm{~cm}$ (Figura $2 C$ ), por lo que se recortaron los bordes para acoplarlo y se colocaron tres anclas bioabsorbibles de $2.0 \mathrm{~mm}$ en los vértices de la lesión. Por último, se realizaron microperforaciones, se insertó el fragmento condral en la rótula, el cual se sujetó con las suturas de las
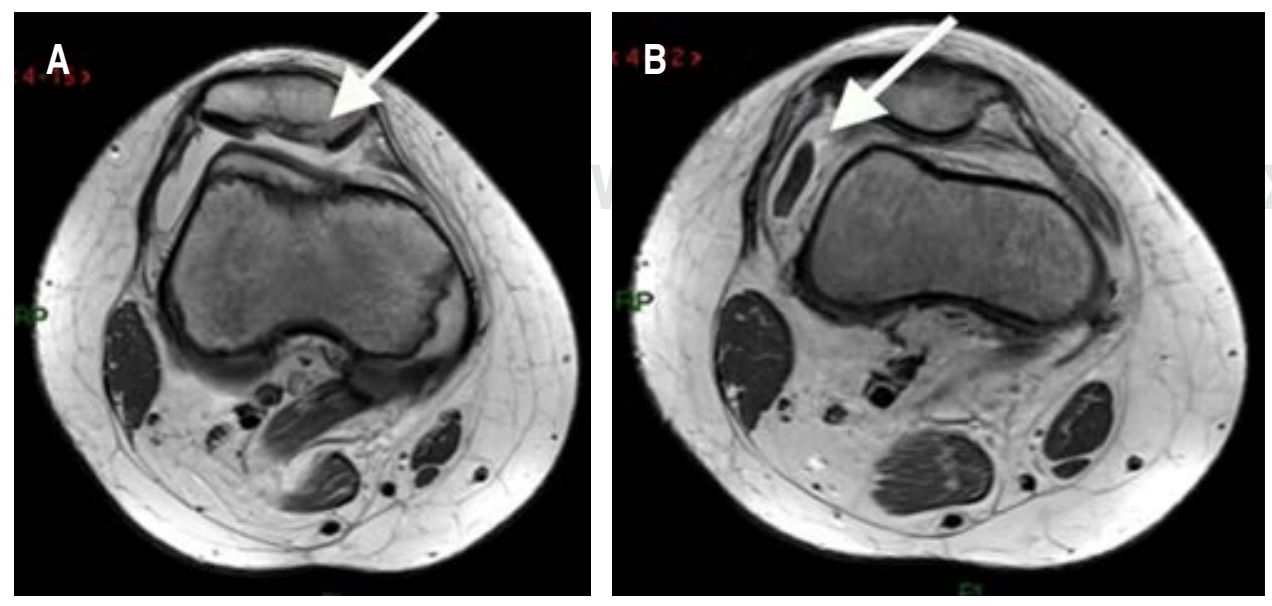

Figura 1:

Resonancia magnética de rodilla derecha que evidencia: (A) lesión condral rotuliana, lesión de ligamento patelofemoral media y rótula lateralizada en secuencia T1 y (B) fragmento condral libre en receso medial. 

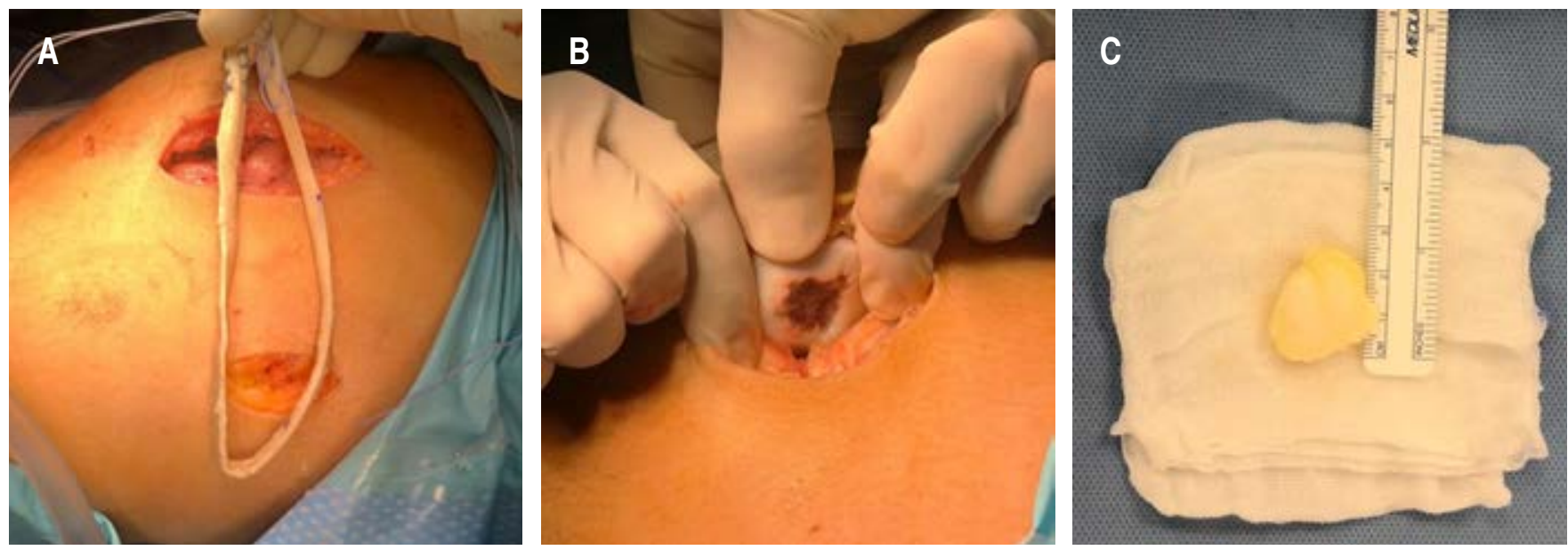

Figura 2: Imágenes quirúrgicas que muestra (A) reparación de ligamento patelofemoral medial. (B) Lesión condral en rotula. (C) Fragmento condral libre.

anclas, y se colocó un coágulo obtenido vía intravenosa entre el hueso y el cartílago (Figura 3). El paciente se dio de alta al segundo día postquirúrgico con rodillera articulada; se difirió el apoyo por un mes. Finalmente, la terapia física inició una semana después de la cirugía con dos sesiones semanales por 12 semanas.

Después de 19 semanas posteriores al procedimiento quirúrgico, el paciente se encontró sin dolor, con arcos de movilidad completos y con discreta sensación de rigidez; maniobra de cepillo positiva debido a las suturas, maniobra de lateralización de rótula con dolor $2 / 10$, marcha independiente no claudicante y fuerza $4 / 5$ al realizar extensión y flexión de la rodilla en comparación con la contralateral. Se realizó una resonancia magnética de control, la cual evidenció la integración del fragmento condral patelar con adecuada congruencia articular (Figura $4 A$ y $B$ ).

\section{DISCUSIÓN}

La reparación condral del caso presentado estuvo basada en la técnica reportada por Pritsch en $1995,{ }^{22}$ de la que se modificó la sujeción ósea por anclas bioabsorbibles. Cabe mencionar que el paciente también presentó una lesión del ligamento patelofemoral medial presente en $76 \%$ de las luxaciones de rótula. ${ }^{23} \mathrm{Se}$ ha reportado que esta última lesión por sí sola puede causar artrosis patelofemoral hasta en $30-50 \%$ de los casos si no se repara quirúrgicamente, debido a un mal acoplamiento entre la rótula y la tróclea, lo que conlleva a un desequilibrio de la transmisión de cargas en un lapso de 12 a 15 años. ${ }^{24,25}$
Existen varios métodos de fijación de lesiones condrales, como la fijación con tornillos metálicos, que han mostrado hasta $90 \%$ de éxito a los tres meses $;{ }^{26}$ sin embargo, se ha reportado, como desventaja, que suele realizarse una perforación de mayor diámetro y se generan lesiones periféricas al tornillo por la fricción de la superficie metálica contra el cartílago en menor frecuencia, como sucede en la fijación con grapas. ${ }^{27}$ La fijación retrógrada ha tenido malos resultados, cuyo porcentaje de éxito es de $38 \%$ por falta de precisión en la colocación de los tornillos. ${ }^{28,29}$

En nuestro caso, decidimos la fijación con tornillos bioabsorbibles, combinado con microfracturas y colocación de coágulo como el tratamiento indicado, ya que las microperforaciones hasta el hueso esponjoso subyacente liberan la sangre con células

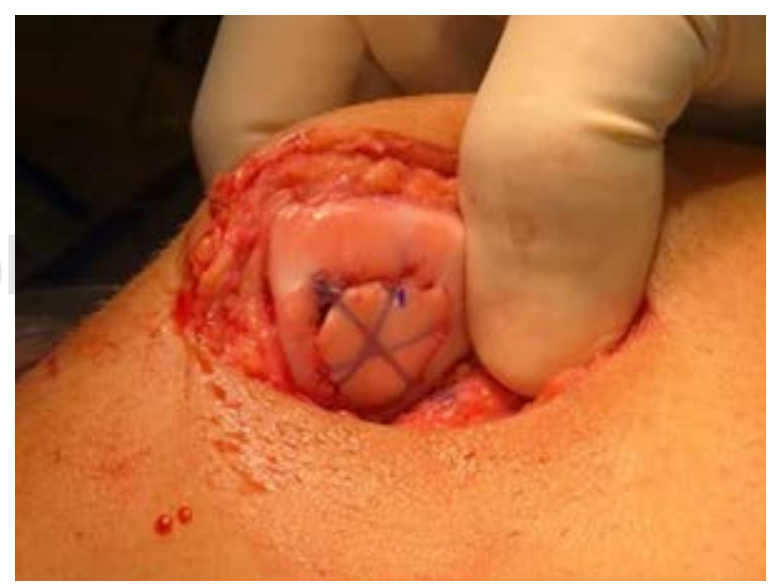

Figura 3: Reparación de la lesión condral. Fragmento condral fijado con tres anclas y con base en el coágulo autógeno. 
Figura 4:

Resonancia magnética postquirúrgica que muestra ( $\mathrm{A}$ y $\mathrm{B}$ ) corte transversal y sagital en secuencia T1 con una adecua-

da integración del fragmento condral.
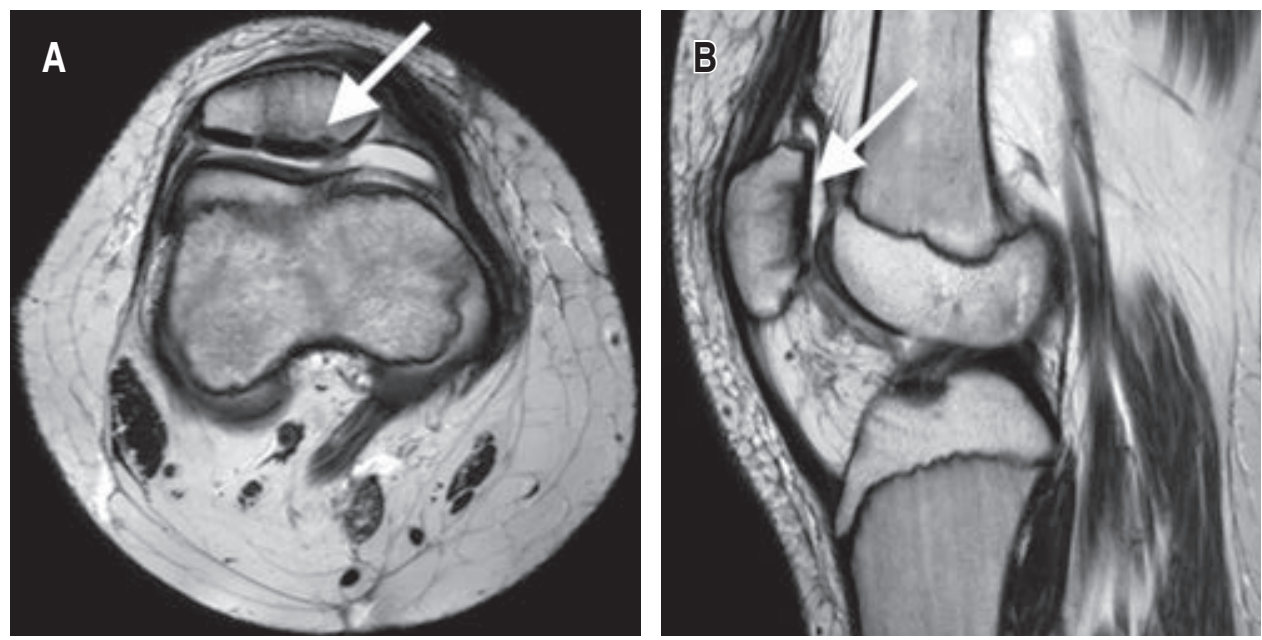

mesenquimales, lo que sirve para reparar el tejido dañado que, junto con la colocación del coágulo, asegurará un lecho para la integración del fragmento condral al hueso. ${ }^{30}$

\section{CONCLUSIÓN}

La técnica de reparación de lesión condral de la rótula mediante anclas bioabsorbibles y coágulo obtuvo un buen desenlace a las 19 semanas. Como desventajas, encontramos su costo y la disponibilidad de las anclas. No obstante, creemos que es necesario realizar más estudios con más pacientes para establecer una mayor precisión en la tasa de éxito.

\section{BIBLIOGRAFIA}

1. Kingma J, Ten Duis HJ. Injuries due to school sports accidents in 4 to 13-years old children. Percept Mot Skills. 2000; 90: 319-325.

2. Oeppen RS, Connolly SA, Bencardino JT. Acute injury of the articular cartilage and subchondral bone: a common but unrecognized lesion in the immature knee. AJR Am J Roentgenol. 2004; 182 (1): 111-117.

3. Vilchis H, Vega EA, Valle G. Lesión osteocondral de patela reparada mediante sutura. Presentación de caso. Rev Mex Ortop. 2008; 22 (1): 50-54.

4. Seeley MA, Knesek M, Vanderhave KL. Osteochondral injury afteracute patellar dislocation in children and adolescents. J Pediatr Orthop. 2013; 33 (5): 511-518.

5. Salzmann G, Niemeyer P, Hochrein A. Articular cartilage repair of the knee in children and adolescents. Orthop $\mathrm{J}$ Sports Med. 2018; 6 (3): 2325967118760190.

6. Kocher MS, DiCanzio J, Zurakowski D. Diagnostic performance of clinical examination and selective magnetic resonance imaging in the evaluation of intraarticular knee disorders in children and adolescents. Am J Sports Med. 2001; 29: 292-296.

7. Tavernier T, Dejour D. Knee imaging: what is the best modality. J Radiol. 2001; 82 (pt 2): 387-405 [en francés].
8. Noyes FR, Stabler CL. A system for grading articular cartilage lesions at arthroscopy. Am J Sports Med. 1989; 17: 505-513.

9. Lohmander LS, Dahlberg L, Ryd L. Increased levels of proteoglycan fragments in knee joint fluid after injury. Arthritis Rheum. 1989; 32: 1434-1442.

10. Camp C, Stuart M, Krych A. Current concepts of articular cartilage restoration techniques in the knee. Sports Health 2014; 6 (3): 265-273.

11. Micheli LJ, Moseley JB, Anderson AF. Articular cartilage defects of the distal femur in children and adolescents: treatment with autologous chondrocyte implantation. J Pediatr Orthop. 2006; 26 (4): 455-460.

12. Alford J, Cole B. Cartilage restoration, Part 1. Am J Sports Med. 2005; 33 (2): 295-306.

13. MacConaill MA. The function of intra-articular fibrocartilages, with special reference to the knee and inferior radioulnar joints. J Anat. 1932; 66: 210-227.

14. Mouzopoulos G, Borbon C, Siebold R. Patellar chondral defects: a review of a challenging entity. Knee Surg Sports Traumatol Arthrosc. 2011; 19 (12): 1990-2001.

15. Alford J, Cole B. Cartilage restoration, Part 2: techniques, outcomes, and future directions. Am J Sports Med. 2005; 33 (3): 443-460.

16. Czitrom AA, Langer F, McKee N. Bone and cartilage allotransplantation: a review of 14 years of research and clinical studies. Clin Orthop Relat Res. 2006; 208: 141-145.

17. Van Jonbergen HP, Poolman RW, van Kampen A. Isolated patellofemoral osteoarthritis. Acta Orthop. 2010; 81 (2): 199-205.

18. Ozturk A, Ozdemir MR, Ozkan Y. Osteochondral autografting (mosaicplasty) in grade IV cartilage defects in the knee joint: 2 to 7 year results. Int Orthop. 2006; 30 (3): 200-204.

19. Sanders TL, Pareek A, Johnson NR. Patellofemoral arthritis after lateral patellar dislocation. Am J Sports Med. 2017; 45 (5): 1012-1017.

20. Cicuttini F, Ding C, Wluka A. Association of cartilage defects with loss of knee cartilage in healthy, middle-age adults: a prospective study. Arthritis Rheum. 2005; 52: 2033-2039.

21. Davies-Tuck ML, Wluka AE, Wang Y. The natural history of cartilage defects in people with knee osteoarthritis. Osteoarthritis Cartilage. 2008; 16: 337-342.

22. Pritsch M, Velkes S, Levy O. Suture fixation of osteochondral fractures of the patella. J Bone Joint Surg Br. 1995; 77-B: 154-155. 
An Med (Mex) 2020; 65 (2): 153-157

23. Kirsch M, Fitzgerald S, Friedman H. Transient patellar dislocation: diagnosis with MR imaging. AJR Am J Roentgenol. 1993; 161 (1): 109-113.

24. Nomura E, Inoue M, Kobayashi S. Long-term follow-up and osteoarthritis change after medial patellofemoral ligament reconstruction for recurrent patellar dislocation. Am J Sports Med. 2007; 35 (11): 1851-1858.

25. Van haver A, De Roo K, De Beule M. The effect of trochlear dysplasia on patellofemoral biomechanics: a cadaveric study with simulated trochlear deformities. Am J Sports Med. 2015; 43 (6): 1354-1361.

26. Cugat R, Garcia M, Cusco X. Osteochondritis dissecans: a historical review and its treatment with cannulated screws. Arthroscopy. 1993; 9: 675-684.
27. Kivisto R, Pasanen L, Leppilahti J. Arthroscopic repair of osteochondritis dissecans of the femoral condyles with metal staple fixation: a report of 28 cases. Knee Surg Sports Traumatol Arthrosc. 2002; 10: 305-309.

28. Schwarz C, Blazina ME, Sisto DJ. The results of operative treatment of osteochondritis dissecans of the patella. Am J Sports Med. 1988; 16: 522-528.

29. Sekiya LC, Fontbote CA, Harner CD. Arthroscopically assisted retrograde fixation of patellar osteochondritis dissecans using fluoroscopic guidance: a case report and technical note. Arthroscopy. 2003; 19: E1-E7.

30. Gantenbein B. Mesenchymal stem cells and collagen patches for anterior cruciate ligament repair. World Journal of Stem Cells. 2015; 7 (2): 521. 